\title{
La prise en charge médicale en fin de vie varie selon les régions
}

PhD Nicole Steck, PhD Claudia Berlin, Prof. Marcel Zwahlen

Institut de médecine sociale et préventive (ISPM) de Berne

\begin{abstract}
En regard du vieillissement de la population en Suisse, les soins médicaux en fin de vie prennent de plus en plus d'importance. Mais de nombreuses questions restent encore sans réponse. Les chercheurs de l'Institut de médecine sociale et préventive (ISPM)* de Berne ont examiné les différences régionales concernant la densité des soins hospitaliers, l'endroit où les personnes meurent et les coûts des soins en fin de vie.
\end{abstract}

Les soins médicaux et infirmiers aux personnes en fin de vie ou «End-of-Life-Care» ont suscité une attention accrue ces dernières années. En 2009, la Confédération et les cantons ont ainsi défini la «Stratégie nationale en matière de soins palliatifs» qu'ils ont mise en œuvre ces dernières années. Par fin de vie, on entend les derniers jours, semaines ou mois précédant le décès. Même si cette période peut concerner n'importe quelle classe d'âge, plus de 80\% des personnes qui meurent en Suisse ont plus de 65 ans. Le Fonds national suisse (FNS) a lancé en 2012 le programme PNR 67 «Fin de vie» [1]. Ce programme a pour objectif de mieux faire comprendre cette phase de la vie et les thèmes qui y sont liés, comme les directives anticipées du patient, l'accès aux soins palliatifs, l'aide au suicide et l'accompagnement

\section{Résumé}

En regard du vieillissement de la population en Suisse, les soins médicaux en fin de vie prennent de plus en plus d'importance. Par fin de vie, on entend les derniers jours, semaines ou mois précédant le décès. En 2012, le Fonds national suisse pour la recherche scientifique a lancé le programme de recherche PNR 67 "Fin de vie», dans le but de mieux comprendre les enjeux que représentent l'accès aux soins palliatifs, I'assistance au suicide et la prise en charge des proches. Le Professeur André Busato, spécialiste en recherche sur les soins, décédé il y a trois ans, a initié différents projets au sein de I'Institut suisse de médecine sociale et préventive (ISPM) à Berne dans le cadre du programme PNR 67. Les résultats des études désormais disponibles montrent qu'il existe de grandes différences entre les régions en ce qui concerne la densité des soins prodigués en fin de vie, le lieu du décès et les coûts occasionnés. Alors que ces différences s'expliquent en partie suivant la région linguistique et l'infrastructure disponible, aucun lien n'a pu en revanche être établi en ce qui concerne les facteurs socio-économiques, les différences se justifiant pour la plupart par les différents types de maladie et par les préférences des patients. Une partie de la variabilité n'étant cependant ni justifiée ni souhaitée, d'autres études seront nécessaires pour mieux en comprendre les tenants et les aboutissants et déterminer les éventuelles mesures à prendre. par les proches aidants. Le chercheur André Busato, décédé en novembre 2013, a initié à l'ISPM de Berne plusieurs projets dans le cadre du PNR 67, dont la plupart sont désormais achevés. Les résultats de ces projets sont présentés ici.

\section{En Suisse romande, les personnes en fin de vie restent plus longtemps à l'hôpital}

L'analyse de la densité des soins prodigués en fin de vie soulève des questions: quelle période précédant la mort est examinée? Comment la densité est-elle définie et quantifiée? Dans le cadre d'un travail systématique, l'équipe End-of-Life de l'ISPM a examiné de quelle manière ces questions avaient été abordées dans la littérature spécialisée [2]. Plus de 1500 articles ont permis d'identifier 58 études ayant analysé et quantifié la densité des soins médicaux en fin de vie. La période examinée varie de 48 heures à douze mois avant la mort. Afin d'évaluer la densité de ces soins, la plupart des études se sont basées sur les hospitalisations, les admissions en soins intensifs et les chimiothérapies. Cependant, ces indicateurs n'ont pas été validés, et une expertise largement étayée fait défaut.

Les chercheurs se sont ensuite penchés sur la situation en Suisse. Notamment sur les différences régionales concernant le nombre de patients mourant à l'hôpital. Pour cela, ils ont utilisé des données de la Statistique médicale des hôpitaux (voir encadré). L'étude s'est intéressée aux patients de plus de 18 ans ayant été hospitalisés pour une période de six mois maximum entre leur admission et leur décès. Au total, les données de 24400 personnes décédées en 2010 ont été analy- 
sées [3]. Au cours des six derniers mois de leur vie, ces patients ont été hospitalisés en moyenne 2,2 fois et ont passé, en tout, presque un mois (29 jours) à l'hôpital. Près d'un tiers ont été transférés au moins une fois en soins intensifs, où ils ont passé en moyenne 39,7 heures. Une analyse de régression multivariable a révélé que les patients atteints d'un cancer restaient plus longtemps à l'hôpital que les personnes admises suite à un accident, une maladie cardio-vasculaire, une infection ou une maladie de l'appareil digestif. Les patients jeunes (19 à 40 ans) et les patients très âgés (plus de 90 ans) ont, en moyenne, été hospitalisés moins longtemps au cours des six derniers mois de leur vie. Les régions linguistiques présentent en outre des différences notables: en Suisse romande, les patients en fin de vie sont restés en moyenne plus longtemps à l'hôpital qu'en Suisse alémanique, à l'exception de la ville de Bâle, où les personnes âgées passent un cinquième de temps en plus à l'hôpital que la moyenne nationale. De manière générale, les différences régionales sont plus importantes pour les personnes âgées que pour les plus jeunes.

\section{Mourir en institutions: là encore des différences régionales}

La plupart des personnes souhaitent mourir chez elles [4]. Une évaluation de l'Observatoire suisse de la santé (Obsan) a cependant révélé qu'entre 2006 et 2011, plus de trois quarts de toutes les personnes décédées sont mortes dans un hôpital ou une institution [5]. L'équipe End-of-Life de l'ISPM a élaboré un conceptcadre répartissant les différences dans les soins médicaux en fin de vie en trois niveaux de déterminants [6]: le niveau macro couvre l'environnement général comme les facteurs socio-économiques et politiques, la politique de santé et le contexte culturel. Le niveau

\section{Groupe de dialogue «Recherche} prioritaire: recherche sur les soins»

Pour le corps médical, la recherche sur les soins constitue un domaine scientifique important porteur d'avenir. Dans un secteur de la santé en pleine mutation (nouveaux modèles de financement et de soins, évolutions démographiques, transferts sectoriels, etc.), il est impératif que la recherche sur les soins bénéficie d'un ancrage académique. Le Fonds national a reconnu cet impératif et a lancé en 2015 le PNR 74 «Smarter Health Care», un programme national prioritaire de recherche sur les soins (http://www.snf.ch/de/fokusForschung/nationale-forschungspro gramme/nfp-74/).

Afin de pouvoir créer les bases scientifiques d'une recherche indépendante dénuée de tout intérêt particulier, la Fédération des médecins suisses (FMH), la Conférence des sociétés cantonales de médecine (CCM) et Newlndex soutiennent le groupe de dialogue «Recherche prioritaire: recherche sur les soins» de I'Institut de médecine sociale et préventive de l'Université de Berne.

A l'instar d'un forum, un groupe de dialogue a pour but de solliciter l'échange d'informations: les représentants des organisations mentionnées et des groupes de recherche discutent régulièrement des travaux en cours et des projets à venir dans le domaine de la recherche sur les soins. Par ailleurs, le groupe de dialogue vise à sensibiliser le corps médical à la recherche sur les soins et à en favoriser l'acceptation, tout en soulignant les avantages concrets de cette recherche pour le corps médical.

Il est ouvert aux propositions en ce qui concerne les sujets à traiter, les questions, les discussions ou les demandes d'informations supplémentaires. La division Données, démographie et qualité (DDQ) de la FMH assure la coordination du groupe de dialogue et se tient à disposition pour tout complément d'information par courriel à ddq[at]fmh.ch ou par téléphone au 0313591111.

méso concerne les caractéristiques et les préférences du personnel médical ainsi que l'offre locale et l'organisation des soins. Le niveau micro se réfère aux caractéristiques et préférences des patients et du personnel infirmier, mais aussi au type et à la gravité de la maladie (voir fig. 1).

Sur la base de ces trois niveaux de déterminants, l'équipe a examiné 41000 cas de décès de patients de plus de

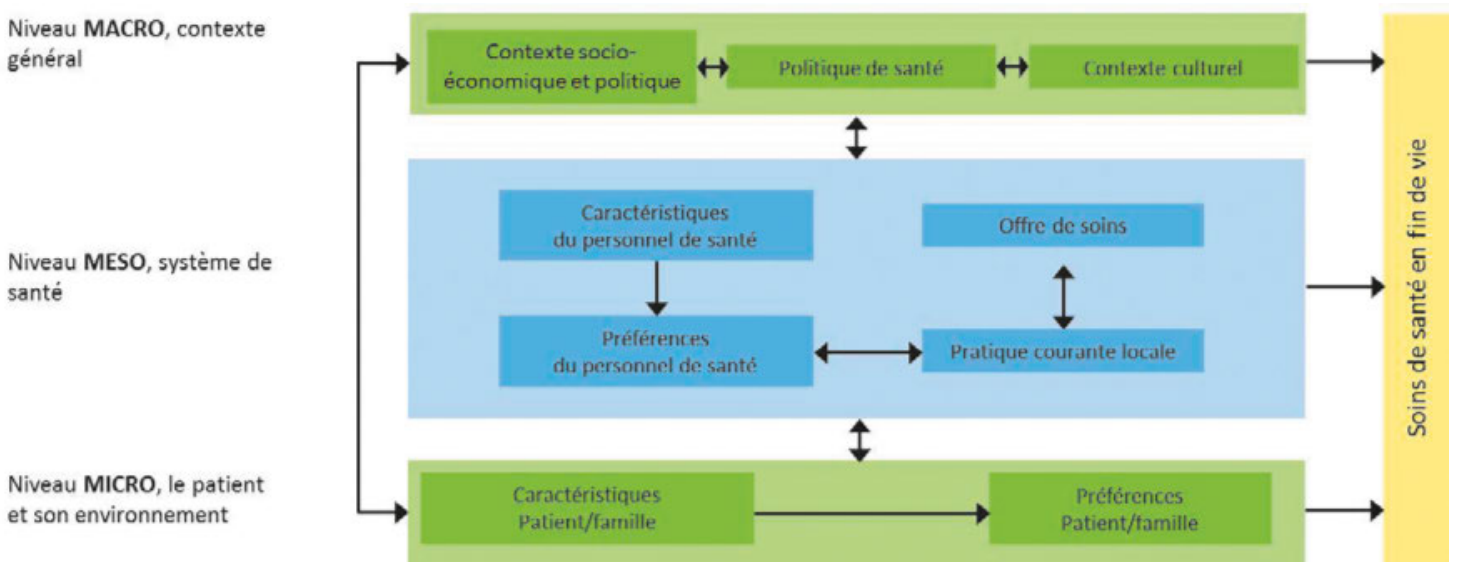

Figure 1: Déterminants possibles pour les différences de soins en fin de vie. 
65 ans dans les institutions suisses et analysé les différences régionales [7], soit environ $80 \%$ de tous les cas de décès chez les plus de 65 ans en 2010. Cette analyse a révélé que la part des décès en institutions augmente avec l'âge pour atteindre presque $80 \%$ chez les plus de 90 ans. Pour les femmes, la probabilité de mourir à l'hôpital est pratiquement de 50\% inférieure à celle des hommes. Mais les caractéristiques individuelles ne sont pas les seuls éléments susceptibles d'influencer le lieu du décès: au plan régional, les personnes habitant à proximité d'une ville ont une plus grande probabilité de mourir à l'hôpital que les personnes résidant en ville même ou à la campagne. Par ailleurs, les personnes de Suisse latine sont plus souvent décédées à l'hôpital que les habitants de Suisse alémanique. Une plus forte densité de médecins et d'institutions de soins ambulatoires est associée à une plus faible probabilité de mourir à l'hôpital.

\section{Les coûts de santé de la dernière année de vie varient considérablement}

Les coûts de santé de la dernière année de vie sont en moyenne cinq fois supérieurs à ceux des années précédentes. Pour examiner les différences en fonction des régions et des fournisseurs de prestations, les chercheurs ont effectué une analyse à petite échelle [8]. Ils ont utilisé les données de six assurances-maladie suisses (CSS, Groupe Mutuel, Helsana, Sanitas, SWICA, Visana), qui représentent environ $60 \%$ des personnes décédées entre 2008 et 2010. Ces données anonymisées ont été reliées à la Statistique des causes de décès de l'Office fédéral de la statistique (OFS) afin d'y ajouter les informations manquantes telles que la cause du décès, la nationalité, l'état civil et la religion. Par ailleurs, la FMH et l'OFS ont mis à disposition d'autres informations concernant les fournisseurs de prestations, leur disponibilité et la population.

L'analyse de toutes les factures traitées par les caissesmaladie pendant les douze derniers mois des 113277 assurés décédés entre 2008 et 2010 a montré que les dépenses médicales en dernière année de vie s'élèvent à 32500 francs en moyenne. Les coûts les plus importants ont été occasionnés par les jeunes patients décédés du cancer (notamment d'une tumeur colorectale, du sein ou de la prostate). La région «la plus chère» affiche des coûts sept fois supérieurs à la région «la moins chère». Ces différences diminuent lorsque divers facteurs sont pris en compte, notamment l'âge, la raison du décès et la région linguistique (voir fig. 2). En revanche, l'offre de soins médicaux dans la région n'a aucun impact sur les coûts. L'étude n'a pas permis d'expliquer toutes les différences de coûts entre les régions, ni celles concernant la dernière année de vie des

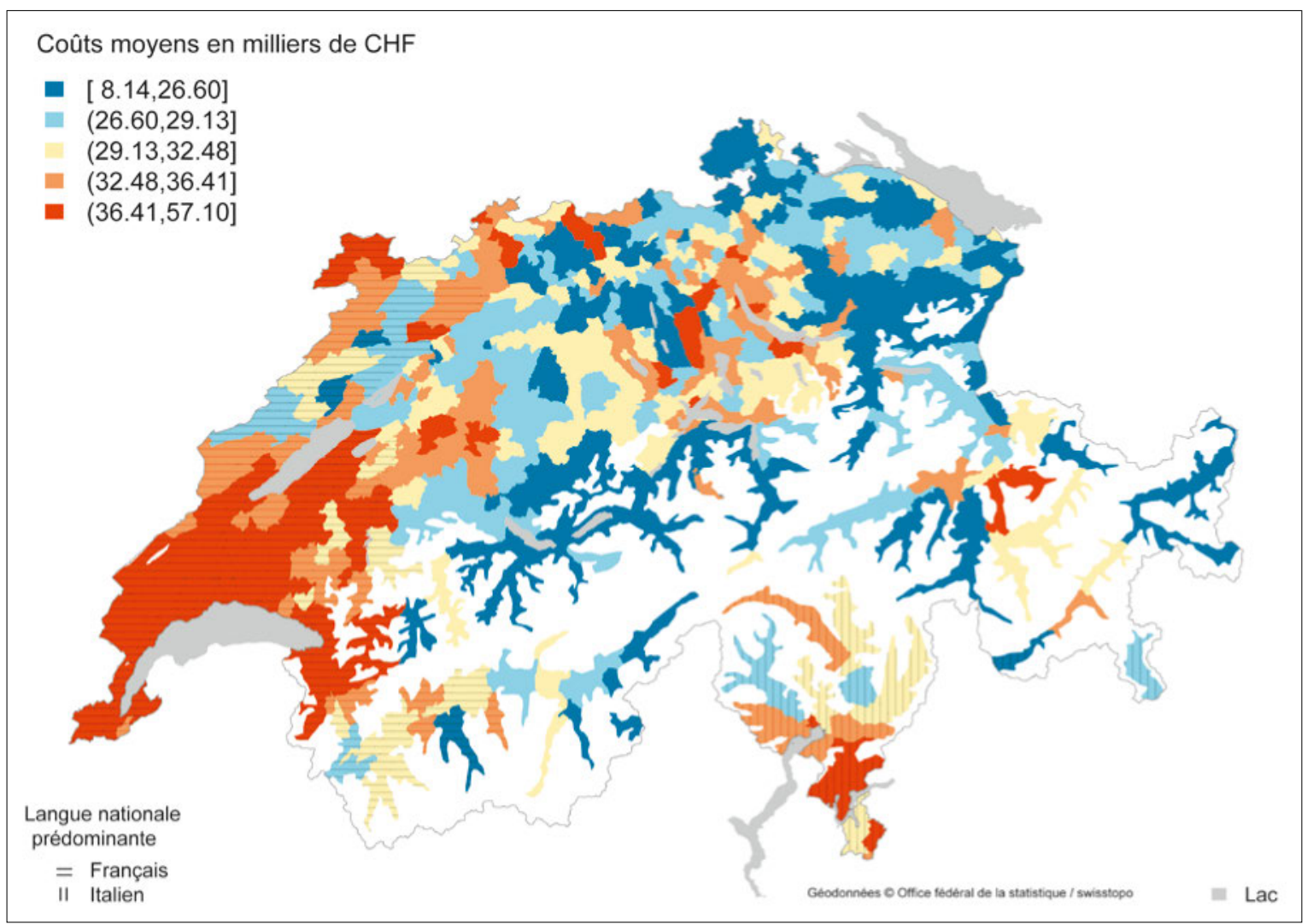

Figure 2: Coûts de santé dans les 12 derniers mois de vie. 
femmes âgées, même après la prise en compte de tous les facteurs. La nette diminution des différences de coûts, lorsqu'on tient compte de la région linguistique, indique que l'organisation des soins en fin de vie est gérée différemment selon les régions en Suisse.

\section{Les personnes seules sollicitent-elles moins d'aide médicale dans leur dernière année de vie?}

Une autre analyse des données des assurances-maladie s'est penchée sur les personnes pour lesquelles il n'existe aucune facture de leur caisse-maladie pour les douze derniers mois de leur vie. L'équipe End-of-Life a examiné dans quelle mesure les caractéristiques personnelles des patients et leur domicile sont déterminants [9] Les patients concernés sont principalement des personnes jeunes, seules, veuves ou divorcées ainsi que des personnes pour lesquelles une maladie relevant de "troubles mentaux et du comportement" (par exemple démence) avait été indiquée comme étant la cause du décès. Il se peut que ces personnes perçoivent moins la nécessité d'une aide médicale, qu'elles sollicitent de l'aide trop tard ou qu'elles soient dépassées par la tracasserie administrative. Dans tous les cas, les raisons de l'inexistence de factures devront faire l'objet d'études supplémentaires.

\section{Les analyses avec des données de routine posent des problèmes}

Toutes les analyses de l'équipe End-of-Life sont basées sur des données qui avaient été recensées à des fins statistiques et administratives au cours des années précédentes. Par conséquent, ces données étaient de qualité variable et n'ont pas permis de répondre à toutes les questions. Des informations importantes comme le degré de sévérité des maladies, les préférences du patient et de ses proches ainsi que la pratique courante n'ont pas été disponibles.

Néanmoins, les études ont montré que la Suisse présentait d'énormes différences régionales pour la dernière phase de vie concernant la densité des soins à l'hôpital, le lieu du décès et les coûts décomptés par les caisses-maladie. La région linguistique et, en partie, l'infrastructure existante permettent d'expliquer quelques-unes des différences, mais de loin pas toutes. Une partie d'entre elles s'explique par des maladies différentes ou les préférences des patients. Mais une autre partie serait injustifiée, et donc indésirable. Pour mieux comprendre les raisons profondes et identifier des contre-mesures éventuelles, des analyses supplémentaires s'avèrent nécessaires.

\section{Statistique médicale des hôpitaux et Statistique des hôpitaux}

La Statistique médicale des hôpitaux recensée par l'Office fédéral de la statistique (OFS) ainsi que la Statistique des hôpitaux sont des sources importantes pour la recherche sur les soins en fin de vie de I'ISPM.

La Statistique médicale des hôpitaux recense chaque année les données des hospitalisations effectuées en Suisse. L'OFS collecte les informations sociodémographiques des patients telles que l'âge, le sexe et la région de domicile, les données administratives comme le type d'assurance et le séjour avant l'admission, et les informations médicales constituées des diagnostics posés et des traitements effectués.

La Statistique des hôpitaux sert principalement à décrire l'infrastructure et l'activité des hôpitaux et des maisons de naissance en Suisse. Les établissements sont tenus de fournir annuellement des données notamment sur leurs prestations ambulatoires et stationnaires, sur leur personnel et sur leurs comptes d'exploitation. Les données collectées sont utilisées à des fins statistiques et administratives.

Crédits photo

(c) Institut de médecine sociale et préventive (ISPM) de Berne

\section{Références}

1 Fonds national suisse. PNR 67 Fin de vie. Secondary PNR 67 Fin de vie 2012. http://www.snf.ch/fr/pointrecherche/programmesnationaux-de-recherche/pnr67-fin-de-vie/Pages/default.aspx

2 Luta X, Maessen M, Egger M, Stuck AE, Goodman DC, Clough-Gorr KM. Measuring intensity of end of life care: a systematic review. PloS One 2015;10(4):e0123764. doi:10.1371/journal.pone.0123764.

3 Maessen M, Panczak R, Luta X, Stuck AE, Egger M, Goodman DC, Zwahlen M, Clough-Gorr KM. The intensity of care during the last six months of life: an analysis of Swiss administrative hospital data. Manuscript under review.

4 Rainsford S, MacLeod RD, Glasgow NJ. Place of death in rural palliative care: A systematic review. Palliative medicine 2016;30(8): 74563. doi: 10.1177/0269216316628779.

5 Obsan. Analyse du lieu de décès 2006-2011. 2013. http://www.bag. admin.ch/themen/gesundheitspolitik/13764/13777/13793/index. html?lang=fr

6 Luta X, Panczak R, Maessen M, Stuck AE, Egger M, Goodman DC, Clough-Gorr KM. Understanding variation in end of life care: A conceptual Framework. Manuscript under review.

7 Luta X, Panczak R, Maessen M, Egger M, Goodman DC, Zwahlen M, Stuck AE, Clough-Gorr KM. Dying among older adults in Switzerland: who dies in hospital, who dies in a nursing home? BMC Palliative Care 2016;15(1):83.

8 Panczak R, Luta X, Maessen M, Stuck AE, Berlin C, Schmidlin K, Reich O, von Wyl V, Goodman DC, Egger M, Zwahlen M, CloughCorr KM. Regional Variation of Cost of Care in the Last 12 Months of Life in Switzerland: Small-Area Analysis Using Insurance Claims Data. Med Care 2016. doi: 10.1097/mlr.0000000000000634.

9 Panczak R, Luta X, Maessen M, Stuck AE, Berlin C, Schmidlin K, Reich O, von Wyl V, Goodman DC, Egger M, Clough-Gorr KM, Zwahlen $M$. Death at no cost? Persons with no health insurance claims in the last year of life in Switzerland. Manuscript under review. 